Neurofeedback, self-regulation, and brain imaging: clinical science and fad in the service of mental disorders

Robert T. Thibault, Michael Lifshitz, Niels Birbaumer,\& Amir Raz

Published in:

Psychotherapy and Psychosomatics 2015; 84: 193-207

doi:

$10.1159 / 000371714$

http://www.karger.com/Article/FullText/371714 


\title{
Neurofeedback, Self-Regulation, and Brain Imaging: Clinical Science and Fad in the Service of Mental Disorders
}

(Neurofeedback: Clinical Science and Fad)

\author{
Robert T. Thibault ${ }^{\text {a }}$, Michael Lifshitz ${ }^{\text {a }}$, Niels Birbaumer ${ }^{\text {b,c }}$, Amir Raz ${ }^{\text {a,d* }}$ \\ a McGill University, 3775 University Street, Montreal, QC, H3A 2B4, Canada \\ ${ }^{\mathrm{b}}$ Institute of Medical Psychology and Behavioral Neurobiology, University of Tübingen, \\ Silcherstrasse 5, D-72076 Tübingen, Germany \\ ${ }^{\mathrm{c}}$ Ospedale San Camillo, Istituto di Ricovero e Cura a Carattere Scientifico (IRCCS), Venezia, \\ Italy \\ ${ }^{\mathrm{d}}$ The Lady Davis Institute for Medical Research, 3755 Cote Ste. Catherine, Montreal, QC, H3T \\ 1E2, Canada
}

*Please address correspondence to:

Professor Amir Raz, 4333 Cote Ste. Catherine, Montreal, QC, H3T 1E4, Canada amir.raz@mcgill.ca

Tel: 1-514-340-8210; Fax: 1-514-340-8124 


\begin{abstract}
Neurofeedback draws on multiple techniques that propel both healthy and patient populations to self-regulate neural activity. Since the 1970s, numerous accounts have promoted electroencephalography-neurofeedback as a viable treatment for a host of mental disorders. Today, while the number of health care providers referring patients to neurofeedback practitioners increases steadily, substantial methodological and conceptual caveats continue to pervade empirical reports. And yet, nascent imaging technologies (e.g., real-time functional magnetic resonance imaging) and increasingly rigorous protocols are paving the road towards more effective applications and better scientific understanding of the underlying mechanisms. Here we outline common neurofeedback methods, illuminate the tenuous state of the evidence, and sketch out future directions to further unravel the potential merits of this contentious therapeutic prospect.
\end{abstract}

Keywords: self-regulation; neuroimaging; rtfMRI; fMRI; EEG; near infrared spectroscopy; psychiatry; mental health; attention deficit hyperactive disorder; neurofeedback. 


\section{INTRODUCTION}

The science and practice of neurofeedback appeals to both researchers and practitioners. Neurofeedback gives real-time graphics or audio that reflect ongoing fluctuations in brain activity (Figure 1). This procedure propels participants to self-regulate otherwise volitionless neural function. The present review focuses on neurofeedback as a means to treating a variety of behavioral disorders (not the large corpus of literature dedicated to the development of braincomputer interfaces (BCIs) for immobilized patients [1]). Despite general enthusiasm, neurofeedback has yet to formally transition from the experimental lab to the larger clinical milieu. Here we review the collective evidence concerning neurofeedback research: our account highlights the relative merits and current shortcomings of the field and charts a future path towards inclusion in the clinical armamentarium.

*** Insert Figure 1 around here***

Neurofeedback developed from biofeedback in the 1960s and now draws on diverse imaging methods to help drive volitional control over electric, magnetic, and hemodynamic fluctuations in brain activity. Following the proliferation of biofeedback techniques targeting physiological parameters (e.g., skin conductance, heart rate, and blood pressure) [2], scientists began to investigate whether individuals could modify their electroencephalographic (EEG) brain signals. Researchers termed the subset of biofeedback techniques strictly concerned with altering brain signatures "neurofeedback". The terms neurofeedback and EEG-biofeedback were once interchangeable. However, the proliferation of new methods for imaging the living human brain has vastly expanded the scope of neurofeedback, which today embraces novel techniques such as functional magnetic resonance imaging (fMRI), functional near infrared spectroscopy (fNIRS), and magnetoencephalography (MEG). Within each imaging modality, moreover, 
researchers have developed distinct neurofeedback protocols that target different brain signals and concomitant physiological processes [3]. Whereas neurofeedback proponents sometimes lump together these diverse protocols, the findings support some techniques more than others. While high-profile journals focus on nascent feedback modalities and often disregard older techniques, publications on EEG-neurofeedback (EEG-nf) continue to outnumber reports on all other neurofeedback techniques combined (Figure 2). Here, we conducted a comprehensive literature search using Web of Science $e^{\mathrm{TM}}$, Scopus, and Google Scholar with the query (neurofeedback OR biofeedback OR real-time) AND (EEG OR electroencephalograph* OR fMRI OR “functional magnetic resonance imaging” OR MEG OR magnetoencephalograph* OR fNIRS OR “near-infrared spectroscopy"). We include the most oft-cited and influential papers concerning each feedback modality. The present review illuminates the tenuous nature of much of the evidence surrounding EEG-nf and explores the potential merits of other, newer feedback techniques.

\footnotetext{
***Insert Figure 2 around here***
}

\section{EEG NEUROFEEDBACK}

When neurofeedback emerged in the 1960s, EEG was the only non-invasive device available to image the living human brain. Today, EEG remains the most common form of neurofeedback. Organizations such as The Biofeedback Certification International Alliance accredit EEG-nf practitioners while The International Society for Neurofeedback \& Research publishes journals centering on EEG-nf (i.e., the Journal of Neurotherapy from 1995 to 2013 and NeuroRegulation since 2014). This trend further extends to clinicians, who sometimes refer patients to neurofeedback practitioners offering expensive and time-consuming treatment 
regimens (see Table 1). Over dozens of sessions, participants develop implicit techniques to alter real-time representations of electrical fluctuations originating from their brain.

*** Insert Table 1 around here***

EEG-nf appears to effectively treat a range of psychological and neurological disorders [4-6]; however, it remains unclear how much the feedback itself as opposed to nonspecific, placebo-like, factors mediate clinical improvements. While researchers originally leveraged EEG-nf to treat a narrow range of ailments including stress, epilepsy, and hyperkinesia, some now claim that this technique can improve a wide variety of disorders including developmental disabilities, traumatic brain injury, stroke, alcoholism, autism spectrum disorder, depression, insomnia, migraines, and chronic pain [4]. To date, however, only one double-blind study has documented greater clinical improvement in patients receiving veritable EEG-nf (i.e., contingent on a signal of interest in their own brain) compared to sham-feedback (i.e., contingent on an unrelated signal from their own brain, activity from a different brain, or a random signal) [7]. Although EEG-nf researchers have reported post-training changes in objective measures including task performance, quantitative EEG [8-10], and resting-state fMRI [11,12], these effects may result from nonspecific variables including expectation, demand characteristics, and contextual factors such as sitting attentively for extended periods of time- - a behavior known to modulate brain function $[13,14]$. Indeed, nonspecific factors often propel very specific changes in both behavior and neural activity [15]. Here we present and evaluate the research supporting EEG-nf as a clinical treatment. EEG-nf may well be an efficacious treatment; however, a close look at the collective literature challenges the tacit assumption that improvement is mostly attributable to the specific feedback of electrical potentials. 
Few EEG-nf experiments employ appropriate control conditions to determine whether feedback itself accounts for the clinical improvement. Many experiments use either no control group or a control condition that differs significantly from the target intervention in terms of length, intensity, and mode of training. And yet, neurofeedback may be particularly conducive to placebo effects because the training proceeds over dozens of sessions [16], involves expensive equipment [17], carries the allure of brain science [18], poorly blinds participants from experimenter expectation [19], and often aims to improve psychological rather than physiological conditions [20-22].

In the classical clinical trial, researchers randomly assign participants to one of two groups: one group receives veritable treatment (e.g., a drug or contingent neurofeedback) while the other group receives a seemingly similar treatment, yet one that contains no active element that is expected to affect physiology (e.g., a placebo pill or non-contingent feedback). Such trials are almost always double-blind (i.e., neither patients nor researchers are privy to group assignment). The difference in improvement between the two groups establishes the treatment effect - how much the drug itself, or the veritable feedback itself, actually improves a given condition. The changes observed in the sham treatment group encompass factors unrelated to the designated treatment mechanism. In EEG-nf studies, however, researchers rarely blind participants and seldom apply non-contingent feedback. Without a sham control group researchers cannot establish the degree to which the designated mechanism is responsible for patient improvement.

Whereas some EEG-nf proponents claim that randomized placebo control trials are impractical for neurofeedback experiments because they are complex and lengthy [23] let alone carry a non-negligible likelihood of breaking the blind [24], recent studies confirm the feasibility 
of rigorous sham-feedback controlled studies [7,25,26]. A few single-blind experiments comparing neurofeedback to control treatments such as electromyography (EMG) biofeedback $[27,28]$ and sham EEG-nf $[29,30]$ demonstrated greater improvements in both healthy and patient populations in the veritable EEG-nf group. In contrast, recent studies showed that EEG-nf and EMG biofeedback were comparable for chronic pain [31], stroke rehabilitation [32], and ADHD [33]. Additional studies concerning pediatric ADHD have used stimulant medication as an active control while attempting to maximize expectancy in both groups by allowing the parents of the affected children to select between medication and neurofeedback [34-36]. While participants in these experiments improved comparably regardless of treatment, a recent study randomly assigned participants to stimulant or neurofeedback treatment and revealed a greater effect in medicated participants [37]. Comparing a new therapy to a standard and accepted treatment is commonplace in clinical research and can provide clinical evidence for treatment efficacy. Nonetheless, only a placebo group (i.e., sham-feedback control) can isolate specific factors.

Notably, six of seven double-blind, sham-controlled studies reported an absence of posttraining behavioral difference between sham-feedback and veritable feedback groups [7,25,26,38-41]. This outcome suggests that the benefits of EEG-nf may rely heavily on nonspecific factors, including those associated with participating in research or meeting with a clinician, rather than on feedback per se. Yet, some EEG-nf proponents argue that the doubleblind, sham-controlled studies fail to provide a real neurofeedback treatment to either group [5]. They argue that such experimental designs may impinge on the effectiveness of neurofeedback by informing participants that they might receive sham-feedback [23] and by preventing clinicians from manually adjusting reward thresholds for each individual [5]. Without tailored 
feedback and assurance of veritable treatment, participants in both sham and veridical-feedback groups may never develop a sense of control over their brainwaves and may, in turn, benefit only from nonspecific factors. Thus, the double-blind sham-controlled studies to date have yet to confirm EEG-nf as either placebo- or feedback-driven. Alas, while EEG-nf does alter both brain patterns and behavioral measures, the current literature does not support a direct connection between the specific feedback and the observed alterations.

\section{Theta/Beta and SMR training}

One of the first and now the most common clinical EEG-nf protocol, theta/beta training, emerged from a serendipitous finding that linked neurofeedback to seizure resistance in cats [42]. Researchers have since extended this protocol to human patients suffering from a variety of psychological disorders. A seminal paper demonstrated that a food-reward neurofeedback paradigm taught cats to down-regulate $12-15 \mathrm{~Hz}$ electrical activity over the sensorimotor cortex [42]. The authors identified this neural signature as a hallmark of attentive immobile states and coined the term sensorimotor rhythm (SMR) to describe this pattern of activity [43]. Shortly thereafter, this research group reported that the cats who had undergone SMR training expressed delayed seizure onset when administered an epileptogenic compound [44]. SMR training soon expanded to human epileptics [45] and ADHD patients [46], both of whom demonstrated clinical improvements. Based on research associating increased theta amplitude with epileptiform activity and attention deficits [47], SMR training later developed into theta/beta training, which rewarded patients for increasing the SMR $(12-15 \mathrm{~Hz})$ or another subset of the beta bandwidth $(12-21 \mathrm{~Hz})$ while simultaneously decreasing theta activity $(4-8 \mathrm{~Hz})$ [48]. Patients undergoing theta/beta training also improved, although no experiment directly compared the effects of SMR to theta/beta training. While theta/beta training continues to dominate the neurofeedback 
literature today, researchers and practitioners have developed additional EEG-nf protocols that train different combinations of theta, alpha, and beta waveforms, as well as brain electronegativity [49] (i.e., slow cortical potentials (SCPs)), isolated brain regions [50], or any deviation from a normalized brain [51,52] (Table 2).

Theta/beta training is the only EEG-nf technique that researchers have tested for specificity. A few EEG-nf proponents derived contentious conclusions from studies in which experimenters reversed reward contingency as a sham comparator (i.e., receiving the same positive feedback for producing exactly the opposite brain activity). If symptoms worsen, then the specific feedback likely accounts for behavioral changes; alternatively, if patients improve similarly regardless of the feedback received, nonspecific factors likely mediate clinical effects. The claim that improved symptomatology depends on specific reward contingencies hinges on one case study of ADHD [46] and two experiments with eight epileptics [48,53]. These experiments used a 'veritable-sham-veritable' design. Over the course of three months, patients first trained to increase theta/beta ratio (veritable condition), then to decrease the same ratio (inverse-sham condition), and finally to increase it once again. Data from these oft-cited studies, dating back to the late 1970s, scantily substantiate the reported conclusions. In one study, three patients had fewer seizures when receiving veritable feedback compared to inverse-sham information; however, five patients experienced more seizures in the veritable feedback condition [53]. In the other experiment, five of eight patients improved with veritable theta/beta training and four of eight improved in the sham-inverse condition [48]. Training was successful only in that, on average, patients reported decreased seizures after all training was complete. In contrast to the authors' interpretations, therefore, these data do not support the specificity of theta/beta training. 
Examining data for clinical conditions such as ADHD and epilepsy, neurofeedback seems to work but perhaps through untargeted processes. A few meta-analyses and reviews promote clinical theta/beta training; however, the majority of included studies are not designed to test specificity. One meta-analysis of theta/beta training for ADHD claimed that EEG-nf is "efficacious and specific" [54], yet not one of the fourteen included studies employed a shamfeedback control group. Instead, the studies used stimulants, wait-listed controls, EMG feedback, group therapy, or no control group. Moreover, most experiments do not blind participants or researchers. Five years later, a follow-up review [5] cited new literature leveraging semi-active, active, and placebo control groups. While the authors presented findings that promote the efficacy of neurofeedback compared to muscle biofeedback, medication, and computerized training, they noted that sham-feedback studies had yet to confirm the importance of contingent feedback. Another review concluded EEG-nf is "probably efficacious" for pediatric ADHD [55]. Of the 14 studies included, eight had not undergone peer-review, two used wait-list controls, two employed group therapy, one had no control, and only one used sham-feedback. Moreover, the single sham-feedback study reported that both contingent and non-contingent feedback comparably improved ADHD symptomatology [56]. Regarding epilepsy, a recent meta-analysis claimed that EEG-nf is "efficacious and specific" for decreasing seizure occurrence [6]. Of the ten studies included, however, three conduct multiple treatments without isolating the effects of EEG-nf and three state that placebo effects drove clinical outcomes. Thus, the collective evidence indicates that theta/beta training offers an efficacious, yet nonspecific, treatment for both ADHD and epilepsy.

Conceptually, confusion lingers as to the use of basic concepts such as the SMR. Since 1981, no published EEG-nf experiment has reversed reward contingency, yet many researchers 
and practitioners continue to assert that the type of feedback specifically determines treatment outcomes [4,57]. The SMR is defined as a signature of attentive, motor inhibiting states [43] the same states assumed throughout EEG-nf. Sitting still for long training periods likely improves motor inhibition, which in turn, may increase SMR amplitude. Thus, theta/beta training may teach participants to regulate cortical activity through nonspecific factors. Notably, the literature also lacks evidence demonstrating parallels in post-training EEG fluctuations and clinical improvement.

\section{Other EEG-nf protocols}

Some researchers and practitioners focus on EEG-nf protocols concerned with selfregulation of brain signals unrelated to theta/beta training. The second most popular technique aims to train brain electronegativity or electropositivity via slow cortical potentials (SCPs). The neurophysiological basis of SCP is much better described than most other EEG signatures [58]. Some studies report that SCP neurofeedback can reduce seizure frequency $[24,59]$ and, after extensive training, allow locked-in patients to communicate through a BCI [60]. Yet, relevant studies share many of the same methodological shortcomings as experiments on theta/beta training and a recent review concluded that specificity remains unsupported ${ }^{47}$.

Over the past decade, researchers popularized upper, or "peak", alpha training to improve cognitive performance $[61,62]$. While training propelled cognitive improvement in many participants, studies lacked active control groups, and instead employed wait-list controls [63], controls who sat passively during feedback sessions [64], or no controls at all [65-67].

Moreover, some studies divided participants into responders and non-responders, thus inflating results $[64,66]$. Interestingly, only individuals with a lower baseline alpha frequency, constituting 70 percent of participants, successfully increased alpha power. As often observed in 
psychological therapies [68], neurofeedback may affect some individuals more than others.

Future experiments that pre-screen participants and successfully select a responsive sample may bolster the evidence promoting upper alpha training. While proponents of this technique have commented that the range of methodologies used to train upper alpha activity makes doubleblind sham-controlled studies prohibitively difficult [69], researchers have already successfully conducted such studies (Egner et al., 2002). Some researchers further argue that individuals must train personalized frequency bands or else risk potential cognitive detriment [61]. Such claims, however, largely rely on case studies and anecdotal evidence [70,71].

A handful of other EEG-nf protocols attempt to improve particular disorders or behavioral traits, but few of the relevant experiments have employed adequate control groups while blinding both participants and experimenters. For example, low resolution electromagnetic tomography (LORETA) neurofeedback aims to train isolated brain regions to improve intelligence [50]; increasing both alpha and theta activity (theta + alpha training) aims to treat alcoholism [72]; and increasing theta relative to alpha (theta/alpha training) supposedly enhances creative performance [73] (Table 2). See Gruzelier, 2013 [62] for a review of performance optimization neurofeedback. Finally, a few contentious commercial applications undercut the credibility of EEG-nf. Live-z score training and the low energy neurofeedback system (LENS) aim to normalize EEG waveforms in relation to a database of resting-state recordings from healthy controls. These techniques lack both a clear physiological basis and support from the mainstream neurofeedback community.

*** Insert Table 2 around here***

After 50 years of research, EEG-nf has largely failed to breakthrough into routine clinical care. The various forms of electrical feedback appear to lend participants mild control over select 
brain signatures, while therapeutic effects seem to derive largely from nonspecific factors. These conclusions encourage novel research directed at teasing apart the nonspecific factors, which contribute to improvement, as well as the circumstances and mechanisms that subserve them. Researchers and clinicians would be able to leverage such knowledge to foster new and effective treatments to utilize both the specific and nonspecific facets of EEG-nf training.

\section{EMERGING NEUROFEEDBACK TECHNIQUES}

Until about a decade ago, EEG was the only available neurofeedback modality. In recent years, however, novel imaging techniques and increased computational power have offered researchers new forms of feedback that draw on diverse brain signals reflecting various underlying neural processes (Figure 3).

Current neurofeedback modalities fall into two broad categories: electromagnetic and hemodynamic training. Whereas EEG and MEG record electromagnetic activity originating from pyramidal cells in superficial cortical regions, fMRI and fNIRS measure local oxygenated and deoxygenated blood concentrations correlated with ongoing neural activity. EEG and MEG have poor spatial resolution but millisecond temporal resolution; fMRI has millimetric spatial resolution yet poor temporal resolution; and fNIRS has poor resolution both spatial and temporal. These intrinsic differences lend select imaging methods to particular applications. For example, because seizure onset alters electrical brain patterns, some researchers promote EEG-nf training as a tool to inhibit pre-epileptic brain states and prevent seizure occurrence [6]. Alternatively, conditions associated with alterations in deep brain functions (e.g., chronic pain and the anterior cingulate cortex) may benefit from imaging methods unrestricted by depth, such as fMRI [74]. 
Examining such strengths and weaknesses may help researchers apply appropriate neurofeedback techniques to specific applications.

Feedback methods also differ in feasibility and availability (Table 1). Researchers and practitioners can quickly learn to conduct EEG-nf with a modest budget using a variety of commercially available software packages and hardware systems. Alternatively, fMRI- and MEG-nf are available to fewer researchers because single imaging sessions cost over $\$ 500$ per hour and because such imaging methods require dedicated state-of-the-art facilities typically found in large institutions rather than in smaller groups. MEG- and fNIRS-nf, moreover, demand considerable technical expertise because no standard or off-the-shelf software packages are available to facilitate these novel, computation-heavy, protocols.

As novel neurofeedback modalities developed from EEG-nf, researchers acknowledged earlier experimental shortcomings and attempted to apply more robust designs to better decipher specific effects. Crucially, in contrast to the classical neurofeedback literature, newer studies tend to employ sham-feedback control groups. Furthermore, nascent neurofeedback techniques may generate smaller placebo effects because they require fewer sessions, in turn, reducing demand characteristics, interactions with a healer, and treatment intensity. While some researchers might argue that longer trials would reduce placebo effects - which are sometimes viewed as short and transient - recent evidence demonstrates that benefits from nonspecific treatments can persist for long durations, from weeks to years $[20,75,76]$. The short duration of training also obviates ethical concerns regarding prolonged sham treatment. On the flip side, most cognitive interventions exhibit strong dose-effect relationships - thus, longer trials may intensify both specific and non-specific effects of neurofeedback. The emerging wave of 
neurofeedback research strives to advance the field in terms of both training efficacy and scientific rigor.

$$
\text { *** Insert Figure } 3 \text { around here*** }
$$

\section{fMRI}

FMRI-nf, often referred to as real-time fMRI (rtfMRI), outputs ongoing data concerning blood oxygen concentrations from any region in the brain. In 2005, deCharms et al. [74] published an fMRI-nf study that employed a careful design and reported robust findings, sparking enthusiasm for this seemingly promising technique. The experiment demonstrated that chronic pain patients who received veritable feedback could learn to modulate rostral anterior cingulate cortex (ACC) activity. Moreover, patients could continue to down-regulate the ACC in the absence of feedback and these neural deactivations paralleled decreases in subjective pain. Such effects were absent in control participants who received feedback from a different brain region or the brain of another participant, or who performed without neurofeedback. This wellcontrolled study remains the strongest piece of evidence supporting fMRI-nf as an effective tool for self-regulating the brain and improving clinical conditions. However, the impact of this one promising study has become shrouded by decade-long skepticism; question marks have turned into exclamation points after a string of independent replication efforts, including by the original authors, was unable to corroborate the reported findings [77,78].

In the aftermath of this problematic study by deCharms et al. [74], most fMRI-nf accounts seldom probe behavioral change but rather report proof-of-concept experiments demonstrating that participants can alter blood flow to select cortical regions. All but two $[79,80]$ of the dozens of published fMRI-nf studies suggested that participants can learn to modulate brain hemodynamics within 40 minutes. Eight fMRI-nf experiments employed sham-feedback 
controls and demonstrated that participants increased their ability to modulate a particular brain region throughout training [81-90]. In contrast, the dozens of remaining studies lacked necessary controls or appropriate analyses to specify neurofeedback as the primary determinant of the observed brain alterations. Indeed, a recent report demonstrated that participants receiving shamneurofeedback expressed increased activation of bilateral insula, ACC, motor areas, and prefrontal areas [91]. Notably, these are the four most common regions trained in fMRI-nf experiments. Thus, only designs that include a sham-feedback control condition can isolate the specific benefits of fMRI-nf.

While EEG-nf takes dozens of sessions to alter electrical activity, fMRI-nf often allows individuals to selectively modify cortical blood flow within a single half-hour session. In fact, participants in some fMRI-nf studies can modulate their brain in the first trial [92-96]. Thus, experimental designs that lack a no-feedback or sham-feedback control cannot dissociate whether the change from baseline relies on feedback or the initial mental strategy. In a yet-to-bepublished fMRI-nf experiment, deCharms targeted this question. He demonstrated that given explicit mental strategies participants successfully learned brain self-regulation, whereas individuals instructed to develop implicit techniques did not [77]. Furthermore, two studies comparing veritable feedback with no feedback [96] or inversely proportional feedback [95] revealed equivalent neural changes from baseline to the first trial. These findings may suggest that initial mental strategies are at least partly responsible for the rapid changes in fMRI signal. Alternatively, a few studies using both humans and animals suggest that explicit techniques are unnecessary and that contingent feedback alone suffices to develop neural regulation $[97,98]$. Hemodynamic learning may occur more quickly because, while the brain lacks receptors that track electrical changes associated with EEG, baroreceptors constantly inform the central 
nervous system about ongoing blood volume relevant to fMRI. While this view remains highly speculative, a series of experiments demonstrating operant conditioning of baroreceptor activation in rats provide preliminary support [99]. Regardless of the cause, the collective evidence indicates that individuals can learn to modulate hemodynamics faster than electrical fluctuations.

Few fMRI-nf studies test if participants can continue to modulate brain activity after the researchers remove feedback. Whereas some experiments demonstrate that participants retain control over target brain regions $[81,86,89,100]$ other studies report no retention $[93,95,101-$ 103]. Thus, while findings suggest that fMRI-nf might help individuals learn to modulate select brain regions, whether participants retain this ability after training remains tenuous.

Whereas fMRI-nf researchers often emphasize potential clinical applications, only sparse accounts have attempted to correlate neurofeedback training with changes in perception or behavior. For example, volitionally dampening ACC activity appears to decrease cigarette cravings $[92,104]$; modulating insular activity affects the perceived valence of emotional stimuli $[82,101]$; altering prefrontal blood flow can improve detection of emotional prosodic intonations [85] and verbal working memory [105]; and training motor areas can improve motor control in Parkinson's patients [100]. While the neurofeedback itself may account for some of these outcomes, many of the relevant experiments would require more robust controls before ascertaining specificity. Both experiments on smoking cessation $[92,104]$ lacked a neutral baseline and instead compared only conditions when participants attempted to enhance craving without neurofeedback versus inhibit craving with neurofeedback. Thus, we cannot conclude whether neurofeedback or mental strategies accounted for the decreased desire to smoke. Furthermore, the experiment on working memory demonstrated that sham-neurofeedback 
enhanced performance on four of the five working memory tasks, even while actually impairing the ability to modulate target brain regions. Neither the emotional valence study with schizophrenics [101] nor the experiment with Parkinson's patients [100] included a shamfeedback control. The Parkinson's patients, moreover, engaged in additional training outside the context of neurofeedback. Finally, the largest fMRI-nf study conducted to date (59 participants) demonstrated that mental rehearsal and neurofeedback treat chronic pain equivalently; yet the report remains unpublished [77]. Thus, while fMRI-nf appears to influence brain activity, applying this technique to modulate specific behaviors requires more rigorous investigation.

\section{fNIRS}

FNIRS probes the same underlying hemodynamics as fMRI [106], yet possesses distinct strengths and weaknesses when applied to neurofeedback. On the one hand, fNIRS is relatively inexpensive, impervious to movement artifacts, and portable throughout daily activities. On the other hand, whereas fNIRS offers spatial resolution on the order of centimeters at surface regions only, fMRI provides millimetric precision throughout the brain. Moreover, unlike the sparse clinical fNIRS literature, an expansive body of research already links fMRI neural signatures with various disorders.

To date, most fNIRS-nf researchers have focused on altering control over surface motor regions. Four recent studies suggested that participants can use fNIRS-nf to increase activity in a variety of motor areas [107-110]. These experiments were particularly susceptible to nonspecific factors because participants can modulate motor cortex activity without neurofeedback - by simply moving a limb or covertly tensing a muscle. In these experiments, participants imagine tapping a finger or clenching a hand. Yet, the researchers omitted testing 
EMGs in the arm to detect covert tensing. Therefore, discreet, possibly subconscious, muscle tension in the arm or hand may have accounted for increases in neural activity.

In two of the four recent fNIRS-nf studies, participants receiving veritable feedback improved modulation over a specific motor region better than sham controls $[107,109]$. In these experiments, contingent feedback appears responsible. The veritable feedback may, however, teach participants to unknowingly increase muscle tension rather than develop new mental techniques. Furthermore, a recent experiment demonstrated that sham-feedback enhances selfregulation of the fNIRS-signal over the motor cortex [111]. The last study lacks a control group [108].

Numerous papers propose fNIRS as a promising modality for use in brain-computer interfaces. The available evidence supports the efficacy of fNIRS-BCIs [112,113]. Yet, this technique does not teach participants to alter brain activity via neurofeedback; rather, individuals simply assume two mental states while the device learns to classify the data. Therefore, fNIRSBCIs are mostly irrelevant to arguments supporting neurofeedback as a mechanism for gaining volitional control over brain dynamics.

\section{MEG}

MEG-nf is still rare, requiring expensive and sparsely available imaging equipment. Similar to EEG, MEG provides a direct measure of neural activity. In contrast to the smearing of EEG signals when crossing the skull and cephalic tissues, however, magnetic fields arrive at the sensors undisturbed. Thus, MEG boasts an impressive spatial resolution and signal-to-noise ratio and may prove especially useful for applications requiring combined spatial and temporal specificity of cortical regions. 
Healthy participants using MEG-nf learned bidirectional control over SMR (often called the mu-rhythm in MEG research) within 64 minutes [114] and over disparate motor cortex activations within 32 minutes [115]. Similar to fNIRS-nf experiments, participants could have unknowingly engaged limb muscles to increase cortical activation. Indeed, stroke patients, who have less control over limb flexion, took much longer-13 to 22 sessions of one to two hoursto gain control over the MEG signal [116]. Furthermore, despite research efforts from several independent groups, completely locked-in patients, without control over body muscles, have been largely unsuccessful in maintaining control over neuroimaging signals [117] — save for one recent case study employing an fNIRS-BCI combined with aversive stimuli [118]. Nonetheless, locked in patients who retain some mobility have learned to communicate via EEG-BCIs [60]. Altogether, therefore, subconscious muscle tension, rather than neurofeedback assisted mental techniques, may account for the improved control over magnetic brain signals. While initial reports sparked enthusiasm, future studies with robust controls would be necessary to confirm MEG-nf as an effective technique to learn brain control.

\section{FUTURE DIRECTIONS}

While neurofeedback has undergone a vigorous revival over the past decade, our review highlights a host of methodological and interpretational caveats that pervade the literature. Although some clinicians already refer patients to practitioners of EEG-nf, the collective findings suggest that this technique scarcely outperforms placebo. The breadth of neurofeedback techniques, however, has greatly expanded with the advent of novel real-time imaging modalities including fMRI, fNIRS, and MEG. Moreover, new studies incorporating increasingly robust experimental designs have begun to unravel the specificity of neurofeedback. And yet, the 
influence of neurofeedback on behavior remains uncertain. Thus, if neurofeedback is to gain a solid footing in clinical practice, it would behoove researchers to focus on demonstrating both specificity and therapeutic outcome.

First, researchers could conduct and replicate well-designed studies to probe whether the feedback itself, rather than placebo factors, account for neural changes. Ideally, these experiments would employ three control groups (i.e., sham-feedback from another participant, feedback from an unrelated brain region, and mental strategy rehearsal) while also collecting subjective data (e.g., what type of feedback participants thought they were receiving, their level of motivation throughout training, how they felt about their performance, and whether they believed the protocol would work). In addition, these studies could implement a double-blind design to minimize the influence investigator knowledge exerts over outcomes. With such designs, researchers could more easily disentangle specific and nonspecific factors. More wellcontrolled studies of this ilk, similar to the landmark experiment by deCharms et al. [74] could bolster evidence to support neurofeedback as a viable tool for brain regulation. Conforming to such stringent methods, one recent study controlled for placebo effects and obtained encouraging results [7].

Second, researchers could aim to demonstrate behavioral or clinical relevance. Since 1996, certification boards have accredited clinical neurofeedback practitioners, yet only one study to date demonstrates greater clinical improvement from EEG-nf compared to shamfeedback [7]. Moreover, few fMRI-nf experiments directly probe therapeutic outcomes. Some clinicians, nonetheless, refer patients to neurofeedback training programs that last more than six months, include as many as 40 sessions, and generally cost between $\$ 4,000$ to $\$ 10,000$ (Table 1). To ensure that patients receive fair treatment for their time and money, future sham controlled 
studies could measure therapeutic outcomes and conduct follow-up sessions. Furthermore, researchers could measure whether differences in neural control parallel changes in behavior. Such findings would support the supposition that brain self-regulation influences clinical behavior.

Developing neurofeedback techniques that target functional connectivity may improve the precision of brain control and engender distinct behavioral results. Researchers have documented altered functional networks in patients with ADHD, depression, schizophrenia, and a host of other disorders [119]. Moreover, collaborative international efforts such as The Human Connectome Project strive to catalog anatomical and functional connectivity throughout the human brain and map out the associated functions. Mounting evidence suggests that many complex behaviours rely on the coordinated activity of multiple regions distributed across functional networks [120]. For neurofeedback, therefore, targeting single regions as opposed to large-scale networks may restrict the ability to hone in on specific behaviors. While networkbased neurofeedback presents particular technical challenges, one research group recently trained participants to modulate connectivity between visual and parietal cortex [94].

Providing a reward contingent on a combination of signals from multiple simultaneous imaging modalities may also increase the effectiveness of neurofeedback. Recently, a proof-ofconcept experiment combined real-time fMRI- and EEG-nf to guide healthy participants to concurrently modulate left amygdala activity and frontal EEG power asymmetry [93]. While such prospects face the same methodological challenges as more conventional forms of neurofeedback, they open new avenues for unlocking the powers of the self-regulating brain.

\section{CONCLUSION}


Scrutinizing relevant findings, we perused the available studies to find evidence largely against the clinical promise of EEG-nf but perhaps with more hopeful prospects for fMRI and other imaging modalities. Regardless of what we found, neurofeedback is in vogue. Pertinent publications abound as feedback techniques continue to advance in parallel with new imaging methods and faster computations. Many researchers and practitioners promote real-time brain feedback as an effective treatment option. Contrary to common opinion, our review of the literature on EEG-nf suggests that treatment outcomes are likely attributable to placebo responses and placebo effects. Moreover, experiments obtaining null-results often shy away from publication, thereby misrepresenting the ratio of negative to positive findings [121]. Mandatory registration of neurofeedback research, as federal drug administrations require for all clinical trials, may obviate this concern, highlight publication bias, and provide data for inclusive metaanalyses. Yet, if EEG-nf consistently outperforms standard treatments requiring comparable investment of time and money, neurofeedback may triumph as a therapeutic option regardless of whether the benefits derive from specific or nonspecific factors. In practice, any patient-therapist interaction draws on psychosocial parameters such as hope, motivation, and expectation. Whereas some clinicians consider EEG-nf as evidence-based medicine and refer patients to practitioners of this dubious technique, newer neurofeedback modalities show more promise. Findings from an increasing number of studies indicate that fMRI-nf may help guide participants toward neural self-regulation. And yet, while almost all fMRI-nf accounts posit feedback as the mechanism underlying learned brain modulation, most experiments shy away from substantiating such claims. Moreover, while proponents vociferously advocate the clinical potential of fMRI-nf, experimenters seldom measure behavioral outcomes and rarely conduct follow-up assessments to verify sustainability. Nascent neurofeedback techniques, including 
fNIRS- and MEG-nf, still need to evolve and amass sufficient evidence to warrant claims promoting their efficacy. Thus, while neurofeedback research continues to flourish, compelling data remain sparse. We hope the present review provides a valuable resource to researchers and clinicians committed to unravelling the intricacies of the self-regulating brain. Adopting a critical lens can only help neurofeedback live up to popular lore and transition to the mainstream clinical milieu. As imaging technologies mature and experimental techniques fine-tune, the exciting field of neurofeedback will either change the way we do mind-body cognitive neuroscience or drown out as a fad. As clinical researchers who examine the available evidence, we dread the latter but earnestly hope for the former. 


\section{ACKNOWLEDGEMENTS}

Dr. Amir Raz acknowledges funding from the Canada Research Chair program, Discovery and Discovery Acceleration Supplement grants from the Natural Sciences and Engineering Research Council of Canada (NSERC), Canadian Institutes of Health Research, and the Volkswagen Foundation (VolkswagenStiftung). Michael Lifshitz acknowledges a Francisco J. Varela

Research Award from the Mind and Life Institute and a Vanier Canada Graduate Scholarship from NSERC. 


\section{REFERENCES}

1. Wolpaw J, Wolpaw EW, editors: Brain-Computer Interfaces: Principles and Practice. New York, Oxford University Press, 2012.

2. Yucha C, Montgomery D, editors: Evidence-based practice in biofeedback and neurofeedback. Wheat Ridge, CO, Association for applied psychophysiology and biofeedback, 2008.

3. Elbert T, Rockstroh B, Lutzenberger W, Birbaumer N, editors: Self-Regulation of the Brain and Behavior. Berlin, Springer-Verlag, 1984.

4. Hammond DC: What is Neurofeedback: An Update. J Neurother 2011;15:305-336.

5. Arns M, Heinrich H, Strehl U: Evaluation of neurofeedback in ADHD: the long and winding road. Biol Psychol 2014;95:108-115.

6. Tan G, Thornby J, Hammond DC, Strehl U, Canady B, Arnemann K, Kaiser DA: MetaAnalysis of EEG Biofeedback in Treating Epilepsy. Clin EEG Neurosci 2009;40:173-179.

7. Ramos-Murguialday A, Broetz D, Rea M, Läer L, Yilmaz O, Brasil FL, Liberati G, Curado MR, Garcia-Cossio E, Vyziotis A, Cho W, Agostini M, Soares E, Soekadar S, Caria A, Cohen LG, Birbaumer N: Brain-machine interface in chronic stroke rehabilitation: a controlled study. Ann Neurol 2013;74:100-108.

8. Leins U, Goth G, Hinterberger T, Klinger C, Rumpf N, Strehl U: Neurofeedback for children with ADHD: a comparison of SCP and Theta/Beta protocols. Appl Psychophysiol Biofeedback 2007;32:73-88.

9. Strehl U, Leins U, Goth G, Klinger C, Hinterberger T, Birbaumer N: Self-regulation of slow cortical potentials: a new treatment for children with attention-deficit/hyperactivity disorder. Pediatrics 2006;118:e1530-e1540.

10. Kropotov JD, Grin-Yatsenko VA, Ponomarev VA, Chutko LS, Yakovenko EA, Nikishena IS: ERPs correlates of EEG relative beta training in ADHD children. Int J Psychophysiol 2005;55:23-34.

11. Lévesque J, Beauregard M, Mensour B: neurofeedback training on the neural substrates of selective attention in children with attention-deficit/hyperactivity disorder: A functional magnetic resonance imaging. Neurosci Lett 2006;301:45-48.

12. Ros T, Théberge J, Frewen PA, Kluetsch R, Densmore M, Calhoun VD, Lanius RA: Mind over chatter: plastic up-regulation of the fMRI salience network directly after EEG neurofeedback. Neuroimage 2013;65:324-335. 
13. Tang Y-Y, Lu Q, Fan M, Yang Y, Posner MI: Mechanisms of white matter changes induced by meditation. Proc Natl Acad Sci U S A 2012;109:10570-10574.

14. Brewer JA, Worhunsky PD, Gray JR, Tang Y-Y, Weber J, Kober H: Meditation experience is associated with differences in default mode network activity and connectivity. Proc Natl Acad Sci U S A 2011;108:20254-20259.

15. Raz A, Michels R: Contextualizing specificity: Specific and non-specific effects of treatment. Am J Clin Hypn 2007;177-182.

16. Kaptchuk TJ, Goldman P, Stone DA, Stason WB: Do medical devices have enhanced placebo effects? J Clin Epidemiol 2000;53:786-792.

17. Waber R, Shiv B, Carmon Z, Ariely D: Commercial features of placebo and therapeutic efficacy. J Am Med Assoc 2008;299:1016-1017.

18. Ali S, Lifshitz M, Raz A: Empirical neuroenchantment: from reading minds to thinking critically. Front Hum Neurosci 2014;27:357.

19. Nichols AL, Maner JK: The Good-Subject Effect : Investigating Participant Demand Characteristics The Good-Subject Effect : Investigating Participant Demand Characteristics. J Gen Psychol 2008;135:151-166.

20. Moseley JB, O’Malley K, Petersen NJ, Menke TJ, Brody BA, Kuykendall DH, Hollingsworth JC, Ashton CM, Wray NP: A controlled trial of arthroscopic surgery for osteoarthritis of the knee. N Engl J Med 2002;347:81-88.

21. Benedetti F, Pollo A, Lopiano L, Lanotte M, Vighetti S, Rainero I: Conscious expectation and unconscious conditioning in analgesic, motor, and hormonal placebo/nocebo responses. J Neurosci 2003;23:4315-4323.

22. Wampold BE, Minami T, Tierney SC, Baskin TW, Bhati KS: The placebo is powerful: estimating placebo effects in medicine and psychotherapy from randomized clinical trials. J Clin Psychol 2005;61:835-854.

23. Brandeis D: Neurofeedback training in ADHD: more news on specificity. Clin Neurophysiol 2011;122:856-857.

24. Kotchoubey B, Strehl U, Uhlmann C, Holzapfel S, König M, Fröscher W, Blankenhorn V, Birbaumer N: Modification of slow cortical potentials in patients with refractory epilepsy: a controlled outcome study. Epilepsia 2001;42:406-416.

25. Lansbergen MM, van Dongen-Boomsma M, Buitelaar JK, Slaats-Willemse D: ADHD and EEG-neurofeedback: a double-blind randomized placebo-controlled feasibility study. J Neural Transm 2011;118:275-284. 
26. Arnold LE, Lofthouse N, Hersch S, Pan X, Hurt E, Bates B, Kassouf K, Moone S, Grantier C: EEG neurofeedback for ADHD: double-blind sham-controlled randomized pilot feasibility trial. J Atten Disord 2013;17:410-419.

27. Bakhshayesh AR, Hänsch S, Wyschkon A, Rezai MJ, Esser G: Neurofeedback in ADHD: a single-blind randomized controlled trial. Eur Child Adolesc Psychiatry 2011;20:481491.

28. Cortoos A, De Valck E, Arns M, Breteler MHM, Cluydts R: An exploratory study on the effects of tele-neurofeedback and tele-biofeedback on objective and subjective sleep in patients with primary insomnia. Appl Psychophysiol Biofeedback 2010;35:125-134.

29. Hoedlmoser K, Pecherstorfer T, Gruber G, Anderer P, Doppelmayr M, Klimesch W, Schabus M: Instrumental conditioning of human sensorimotor rhythm $(12-15 \mathrm{~Hz})$ and its impact on sleep as well as declarative learning. Sleep 2008;31:1401-1408.

30. Pineda JA, Brang D, Hecht E, Edwards L, Carey S, Bacon M, Futagaki C, Suk D, Tom J, Birnbaum C, Rork A: Positive behavioral and electrophysiological changes following neurofeedback training in children with autism. Res Autism Spectr Disord 2008;2:557581 .

31. Glombiewski JA, Bernardy K, Häuser W: Efficacy of EMG- and EEG-Biofeedback in Fibromyalgia Syndrome: A Meta-Analysis and a Systematic Review of Randomized Controlled Trials. Evid Based Complement Alternat Med 2013;2013:962741.

32. Rayegani SM, Raeissadat SA, Sedighipour L, Rezazadeh IM, Bahrami MH, Eliaspour D, Khosrawi S: Effect of neurofeedback and electromyographic-biofeedback therapy on improving hand function in stroke patients. Top Stroke Rehabil 2014;21:137-151.

33. Maurizio S, Liechti MD, Heinrich H, Jäncke L, Steinhausen H-C, Walitza S, Brandeis D, Drechsler R: Comparing tomographic EEG neurofeedback and EMG biofeedback in children with attention-deficit/hyperactivity disorder. Biol Psychol 2014;95:31-44.

34. Rossiter T: The effectiveness of neurofeedback and stimulant drugs in treating AD/HD: Part I. Review of methodological issues. Appl Psychophysiol Biofeedback 2004;29:95112.

35. Monastra VJ, Monastra DM, George S: The effects of stimulant therapy, EEG biofeedback, and parenting style on the primary symptoms of attentiondeficit/hyperactivity disorder. Appl Psychophysiol Biofeedback 2002;27:231-249.

36. Fuchs T, Birbaumer N, Lutzenberger W, Gruzelier JH, Kaiser J: Neurofeedback Treatment for Attention-Deficit / Hyperactivity Disorder in Children : A Comparison With Methylphenidate. Appl Psychophysiol Biofeedback 2003;28. 
37. Ogrim G, Hestad KA: Effects of neurofeedback versus stimulant medication in attentiondeficit/hyperactivity disorder: a randomized pilot study. J Child Adolesc Psychopharmacol 2013;23:448-457.

38. Egner T, Strawson E, Gruzelier JH: EEG signature and phenomenology of alpha/theta neurofeedback training versus mock feedback. Appl Psychophysiol Biofeedback 2002;27:261-270.

39. Gillham S, Wild H, Bayer Z, Mitchell M, Sandberg-Lewis K, Colbert A: Low Energy Neurofeedback System (LENS) for stress, anxiety, and cognitive function: an exploratory study. BMC Complement Altern Med 2012;12:145.

40. Logeman HNA, Lansbergen MM, van Os TWDP, Bocker KBE, Kenemans JL: The effectiveness of EEG-feedback on attention, impulsivity and EEG: a sham feedback controlled study. Neurosci Lett 2010;479:49-53.

41. Vollebregt MA, van Dongen-Boomsma M, Buitelaar JK, Slaats-Willemse D: Does EEGneurofeedback improve neurocognitive functioning in children with attentiondeficit/hyperactivity disorder? A systematic review and a double-blind placebo-controlled study. J Child Psychol Psychiatry 2014;55:460-472.

42. Wyrwicka W, Sterman MB: Instrumental conditioning of sensorimotor cortex EEG spindles in the waking cat. Physiol Behav 1968;3:703-707.

43. Roth SR, Sterman MB, Clemente CD: Comparison of EEG correlates of reinforcement, internal inhibition and sleep. Electroencephalogr Clin 1967;23:509-520.

44. Sterman MB, LoPresti RW, Fairchild MD: Electroencephalographic and Behavioral Studies of Monomethyl Hydrazine Toxicity in the Cat. J Neurother 2010;14:293-300.

45. Sterman M, Friar L: Suppression of seizures in an epileptic following sensorimotor EEG feedback training. Electroencephalogr Clin Neurophysiol 1972;33:89-95.

46. Lubar JF, Shouse MN: EEG and behavioral changes in a hyperkinetic child concurrent with training of the sensorimotor rhythm (SMR): a preliminary report. Biofeedback Self Regul 1976;1:293-306.

47. Matsuura M, Okubo Y, Toru M, Kojima T, He Y, Hou Y, Shen Y, Lee CK: A crossnational EEG study of children with emotional and behavioral problems: a WHO collaborative study in the Western Pacific Region. Biol Psychiatry 1993;34:59-65.

48. Lubar JF, Shabsin HS, Natelson SE, Holder GS, Whitsett SF, Pamplin WE, Krulikowski DI: EEG operant conditioning in intractable epileptics. Arch Neurol 1981;38:700-704.

49. Rockstroh B, Birbaumer N, Elbert T, Lutzenberger W: Operant control of EEG and eventrelated and slow brain potentials. Biofeedback Self Regul 1984;9:139-160. 
50. Cannon R, Lubar J, Congedo M, Thornton K, Towler K, Hutchens T: The effects of neurofeedback training in the cognitive division of the anterior cingulate gyrus. Int $\mathrm{J}$ Neurosci 2007;117:337-357.

51. Ochs L: The Low Energy Neurofeedback System ( LENS ): Theory, Background, and Introduction. J Neurother 2006;10:5-39.

52. Collura TF, Guan J, Tarrant J, Bailey J, Starr F: EEG Biofeedback Case Studies Using Live Z-Score Training and a Normative Database. J Neurother 2010;14:22-46.

53. Sterman M, MacDonald L: Effects of central cortical EEG feedback training on incidence of poorly controlled seizures. Epilepsia 1978;19:207-222.

54. Arns M, de Ridder S, Strehl U, Breteler M, Coenen A: Efficacy of neurofeedback treatment in ADHD: the effects on inattention, impulsivity and hyperactivity: a metaanalysis. Clin EEG Neurosci 2009;40:180-189.

55. Lofthouse N, Arnold LE, Hersch S, Hurt E, DeBeus R: A review of neurofeedback treatment for pediatric ADHD. J Atten Disord 2012;16:351-372.

56. Perreau-Linck E, Lessard N, Lévesque J, Beauregard M: Effects of Neurofeedback Training on Inhibitory Capacities in ADHD Children: A Single-Blind, Randomized, Placebo-Controlled Study. J Neurother 2010;14:229-242.

57. Monastra VJ, Lynn S, Linden M, Lubar JF, Gruzelier J, LaVaque TJ:

Electroencephalographic biofeedback in the treatment of attention-deficit/hyperactivity disorder. Appl Psychophysiol Biofeedback 2005;30:95-114.

58. Birbaumer N, Elbert T, Canavan A, Rockstroh B: Slow potentials of the cerebral cortex and behavior. Physiol Rev 1990;70:1-41.

59. Rockstroh B, Elbert T, Birbaumer N, Wolf P, Düchting-Röth A, Reker M, Daum I, Lutzenberger W, Dichgans J: Cortical self-regulation in patients with epilepsies. Epilepsy Res 1993;14:63-72.

60. Birbaumer N, Ghanayim N, Hinterberger T, Iversen I, Kotchoubey B, Kübler A, Perelmouter J, Taub E, Flor H: A spelling device for the paralysed. Nature 1999;398:297298.

61. Bazanova OM, Vernon D: Interpreting EEG alpha activity. Neurosci Biobehav Rev 2013;44:94-110.

62. Gruzelier JH: EEG-neurofeedback for optimising performance. I: A review of cognitive and affective outcome in healthy participants. Neurosci Biobehav Rev 2014;44:124-141. 
63. Zoefel B, Huster RJ, Herrmann CS: Neurofeedback training of the upper alpha frequency band in EEG improves cognitive performance. Neuroimage 2011;54:1427-1431.

64. Bazanova OM, Balioz N V, Muravleva KB, Skoraya M V: Effect of voluntary EEG $\alpha$ power increase training on heart rate variability. Hum Physiol 2013;39:86-97.

65. Angelakis E, Stathopoulou S, Frymiare JL, Green DL, Lubar JF, Kounios J: EEG neurofeedback: a brief overview and an example of peak alpha frequency training for cognitive enhancement in the elderly. Clin Neuropsychol 2007;21:110-129.

66. Bazanova OM, Mernaya EM, Shtark MB: Biofeedback in psychomotor training. Electrophysiological basis. Neurosci Behav Physiol 2009;39:437-447.

67. Hanslmayr S, Sauseng P, Doppelmayr M, Schabus M, Klimesch W: Increasing Individual Upper Alpha Power by Neurofeedback Improves Cognitive Performance in Human Subjects. Appl Psychophysiol Biofeedback 2005;30:1-10.

68. Lindhiem O, Kolkoa DJ, Cheng Y: Predicting Psychotherapy Benefit: A Probabilistic and Individualized Approach. Behav Ther 2012;43:381-392.

69. Vernon D, Dempster T, Bazanova O, Rutterford N, Pasqualini M, Andersen S: Alpha Neurofeedback Training for Performance Enhancement: Reviewing the Methodology. J Neurother 2009;13:214-227.

70. Hammond DC, Kirk L: First, Do No Harm: Adverse Effects and the Need for Practice Standards in Neurofeedback. J Neurother 2008;12:79-88.

71. Bazanova O, Aftanas L: Individual EEG Alpha Activity Analysis for Enhancement Neurofeedback Efficiency: Two Case Studies. J Neurother 2010;14:244-253.

72. Saxby E, Peniston EG: Alpha-theta brainwave neurofeedback training: An effective treatment for male and female alcoholics with depressive symptoms. J Clin Psychol 1995;51:685-693.

73. Egner T, Gruzelier JH: Ecological validity of neurofeedback: modulation of slow wave EEG enhances musical performance. Neuroreport 2003;14:1221-1224.

74. DeCharms RC, Maeda F, Glover GH, Ludlow D, Pauly JM, Soneji D, Gabrieli JDE, Mackey SC: Control over brain activation and pain learned by using real-time functional MRI. Proc Natl Acad Sci U S A 2005;102:18626-18631.

75. Khan A, Redding N, Brown WA: The persistence of the placebo response in antidepressant clinical trials. J Psychiatr Res 2008;42:791-796.

76. Finniss DG, Kaptchuk TJ, Miller F, Benedetti F: Placebo Effects: Biological, Clinical and Ethical Advances 2011;375:686-695. 
77. Sulzer J, Haller S, Scharnowski F, Weiskopf N, Birbaumer N, Blefari ML, Bruehl a B, Cohen LG, DeCharms RC, Gassert R, Goebel R, Herwig U, LaConte S, Linden D, Luft A, Seifritz E, Sitaram R: Real-time fMRI neurofeedback: progress and challenges. Neuroimage 2013;76:386-399.

78. Birbaumer N, Ruiz S, Sitaram R: Learned regulation of brain metabolism. Trends Cogn Sci 2013;17:295-302.

79. Hampson M, Scheinost D, Qiu M, Bhawnani J, Lacadie CM, Leckman JF, Constable RT, Papademetris X: Biofeedback of real-time functional magnetic resonance imaging data from the supplementary motor area reduces functional connectivity to subcortical regions. Brain Connect 2011;1:91-98.

80. Berman BD, Horovitz SG, Venkataraman G, Hallett M: Self-modulation of primary motor cortex activity with motor and motor imagery tasks using real-time fMRI-based neurofeedback. Neuroimage 2012;59:917-925.

81. Caria A, Veit R, Sitaram R, Lotze M, Weiskopf N, Grodd W, Birbaumer N: Regulation of anterior insular cortex activity using real-time fMRI. Neuroimage 2007;35:1238-1246.

82. Caria A, Sitaram R, Veit R, Begliomini C, Birbaumer N: Volitional control of anterior insula activity modulates the response to aversive stimuli. A real-time functional magnetic resonance imaging study. Biol Psychiatry 2010;68:425-432.

83. Lawrence EJ, Su L, Barker GJ, Medford N, Dalton J, Williams SCR, Birbaumer N, Veit R, Ranganatha S, Bodurka J, Brammer M, Giampietro V, David AS: Self-regulation of the anterior insula: Reinforcement learning using real-time fMRI neurofeedback. Neuroimage 2014;88:113-124.

84. McCaig RG, Dixon M, Keramatian K, Liu I, Christoff K: Improved modulation of rostrolateral prefrontal cortex using real-time fMRI training and meta-cognitive awareness. Neuroimage 2011;55:1298-1305.

85. Rota G, Sitaram R, Veit R, Erb M, Weiskopf N, Dogil G, Birbaumer N: Self-regulation of regional cortical activity using real-time fMRI: the right inferior frontal gyrus and linguistic processing. Hum Brain Mapp 2009;30:1605-1614.

86. Zotev V, Krueger F, Phillips R, Alvarez RP, Simmons WK, Bellgowan P, Drevets WC, Bodurka J: Self-regulation of amygdala activation using real-time FMRI neurofeedback. PLoS One 2011;6:e24522.

87. Yoo S-S, Lee J-H, O'Leary H, Panych LP, Jolesz FA: Neurofeedback fMRI-mediated learning and consolidation of regional brain activation during motor imagery. Int $\mathbf{J}$ Imaging Syst Technol 2008;18:69-78. 
88. Chiew M, Laconte SM, Graham SJ: NeuroImage Investigation of fMRI neurofeedback of differential primary motor cortex activity using kinesthetic motor imagery. Neuroimage 2012;61:21-31.

89. Zotev V, Phillips R, Young KD, Drevets WC, Bodurka J: Prefrontal Control of the Amygdala during Real-Time fMRI Neurofeedback Training of Emotion Regulation. PLoS One 2013;8:e79184.

90. Rota G, Handjaras G, Sitaram R, Birbaumer N, Dogil G: Reorganization of functional and effective connectivity during real-time fMRI-BCI modulation of prosody processing. Brain Lang 2011;117:123-132.

91. Ninaus M, Kober SE, Witte M, Koschutnig K, Stangl M, Neuper C, Wood G: Neural substrates of cognitive control under the belief of getting neurofeedback training. Front Hum Neurosci 2013;7:1-10.

92. Canterberry M, Hanlon CA, Hartwell KJ, Li X, Owens M, LeMatty T, Prisciandaro JJ, Borckardt J, Saladin ME, Brady KT, George MS: Sustained reduction of nicotine craving with real-time neurofeedback: exploring the role of severity of dependence. Nicotine Tob Res 2013;15:2120-2124.

93. Zotev V, Phillips R, Yuan H, Misaki M, Bodurka J: Self-regulation of human brain activity using simultaneous real-time fMRI and EEG neurofeedback. Neuroimage 2013;85:985-995.

94. Koush Y, Rosa MJ, Robineau F, Heinen K, W Rieger S, Weiskopf N, Vuilleumier P, Van De Ville D, Scharnowski F: Connectivity-based neurofeedback: dynamic causal modeling for real-time fMRI. Neuroimage 2013;81:422-430.

95. Sulzer J, Sitaram R, Blefari ML, Kollias S, Birbaumer N, Stephan KE, Luft A, Gassert R: Neurofeedback-mediated self-regulation of the dopaminergic midbrain. Neuroimage 2013;83:817-825.

96. Zhang G, Zhang H, Li X, Zhao X, Yao L, Long Z: Functional alteration of the DMN by learned regulation of the PCC using real-time fMRI. IEEE Trans neural Syst Rehabil Eng 2013;21:595-606.

97. Caria A, Weber C, Brötz D, Ramos A, Ticini LF, Gharabaghi A, Braun C, Birbaumer N: Chronic stroke recovery after combined BCI training and physiotherapy: a case report. Psychophysiology 2011;48:578-582.

98. Koralek AC, Jin X, Long JD, Costa RM, Carmena JM: Corticostriatal plasticity is necessary for learning intentional neuroprosthetic skills. Nature 2012;483:331-335. 
99. Dworkin BR: Hypertension as a learned response: The baroreceptor reinforcement hypothesis.; in Elbert T, Langosch W, Steptoe A, Vaitl D (eds): Behavioral medicine in cardiovascular disorders. Chichester, UK, Wiley, 1988, pp 17-47.

100. Subramanian L, Hindle J V, Johnston S, Roberts M V, Husain M, Goebel R, Linden D: Real-time functional magnetic resonance imaging neurofeedback for treatment of Parkinson's disease. J Neurosci 2011;31:16309-16317.

101. Ruiz S, Lee S, Soekadar SR, Caria A, Veit R, Kircher T, Birbaumer N, Sitaram R: Acquired self-control of insula cortex modulates emotion recognition and brain network connectivity in schizophrenia. Hum Brain Mapp 2013;34:200-212.

102. Hamilton JP, Glover GH, Hsu J-J, Johnson RF, Gotlib IH: Modulation of subgenual anterior cingulate cortex activity with real-time neurofeedback. Hum Brain Mapp 2011;32:22-31.

103. Berman BD, Horovitz SG, Hallett M: Modulation of functionally localized right insular cortex activity using real-time fMRI-based neurofeedback. Front Hum Neurosci 2013;7:111.

104. Li X, Hartwell KJ, Borckardt J, Prisciandaro JJ, Saladin ME, Morgan PS, Johnson KA, Lematty T, Brady KT, George MS: Volitional reduction of anterior cingulate cortex activity produces decreased cue craving in smoking cessation: a preliminary real-time fMRI study. Addict Biol 2012;1-10.

105. Zhang G, Yao L, Zhang H, Long Z, Zhao X: Improved working memory performance through self-regulation of dorsal lateral prefrontal cortex activation using real-time fMRI. PLoS One 2013;8:e73735.

106. Cui X, Bray S, Bryant D, Glover G, Reiss A: A quantitative comparison of NIRS and fMRI across multiple cognitive tasks. Neuroimage 2011;54:2808-2821.

107. Kober SE, Wood G, Kurzmann J, Friedrich EVC, Stangl M, Wippel T, Väljamäe A, Neuper C: Near-infrared spectroscopy based neurofeedback training increases specific motor imagery related cortical activation compared to sham feedback. Biol Psychol 2014;95:21-30.

108. Kanoh S, Susila I, Miyamoto K: The Effect of Neurofeedback Training on Cortical Activity during Motor Imagery Revealed by NIRS and fMRI. Int J Bioelectromagn 2011;13:82-83.

109. Mihara M, Hattori N, Hatakenaka M, Yagura H, Kawano T, Hino T, Miyai I: Nearinfrared Spectroscopy-mediated Neurofeedback Enhances Efficacy of Motor Imagerybased Training in Poststroke Victims: A Pilot Study. Stroke 2013;1-8. 
110. Mihara M, Miyai I, Hattori N, Hatakenaka M, Yagura H, Kawano T, Okibayashi M, Danjo N, Ishikawa A, Inoue Y, Kubota K: Neurofeedback using real-time near-infrared spectroscopy enhances motor imagery related cortical activation. PLoS One 2012;7:e32234.

111. Holper L, Wolf M: Motor imagery in response to fake feedback measured by functional near-infrared spectroscopy. Neuroimage 2010;50:190-197.

112. Sitaram R, Zhang H, Guan C, Thulasidas M, Hoshi Y, Ishikawa A, Shimizu K, Birbaumer $\mathrm{N}$ : Temporal classification of multichannel near-infrared spectroscopy signals of motor imagery for developing a brain-computer interface. Neuroimage 2007;34:1416-1427.

113. Fazli S, Mehnert J, Steinbrink J, Curio G, Villringer A, Müller K-R, Blankertz B: Enhanced performance by a hybrid NIRS-EEG brain computer interface. Neuroimage 2012;59:519-529.

114. Mellinger J, Schalk G, Braun C, Preissl H, Rosentiel W, Birbaumer N, Kübler A: An MEG-based brain-computer interface (BCI). Neuroimage 2007;36:581-593.

115. Lal T, Schröder M, Hill N, Preissl H, Hinterberger T, Mellinger J, Bogdan M, Rosenstiel W, Hofmann T, Birbaumer N, Schölkopf B: A brain computer interface with online feedback based on magnetoencephalography; in : Proceedings of the 22nd International Conference on Machine Learning. Bonn, Germany, 2005.

116. Buch E, Weber C, Cohen LG, Braun C, Dimyan M a, Ard T, Mellinger J, Caria A, Soekadar S, Fourkas A, Birbaumer N: Think to move: a neuromagnetic brain-computer interface (BCI) system for chronic stroke. Stroke 2008;39:910-917.

117. De Massari D, Ruf CA, Furdea A, Matuz T, van der Heiden L, Halder S, Silvoni S, Birbaumer N: Brain communication in the locked-in state. Brain 2013;136:1989-2000.

118. Birbaumer N, Gallegos-Ayala G, Wildgruber M, Silvoni S, Soekadar SR: Direct brain control and communication in paralysis. Brain Topogr 2014;27:4-11.

119. Whitfield-Gabrieli S, Ford JM: Default mode network activity and connectivity in psychopathology. Annu Rev Clin Psychol 2012;8:49-76.

120. Biswal BB, Mennes M, Zuo X-N, Gohel S, Kelly C, Smith SM, Beckmann CF, Adelstein JS, Buckner RL, Colcombe S, Dogonowski A-M, Ernst M, Fair D, Hampson M, Hoptman MJ, Hyde JS, Kiviniemi VJ, Kötter R, Li S-J, Lin C-P, Lowe MJ, Mackay C, Madden DJ, Madsen KH, Margulies DS, Mayberg HS, McMahon K, Monk CS, Mostofsky SH, Nagel BJ, Pekar JJ, Peltier SJ, Petersen SE, Riedl V, Rombouts S a RB, Rypma B, Schlaggar BL, Schmidt S, Seidler RD, Siegle GJ, Sorg C, Teng G-J, Veijola J, Villringer A, Walter M, Wang L, Weng X-C, Whitfield-Gabrieli S, Williamson P, Windischberger C, Zang YF, Zhang H-Y, Castellanos FX, Milham MP: Toward discovery science of human brain function. Proc Natl Acad Sci U S A 2010;107:4734-4739. 
121. Chalmers I, Glasziou P, Godlee F: All trials must be registered and the results published. BMJ 2013;346:f105. 


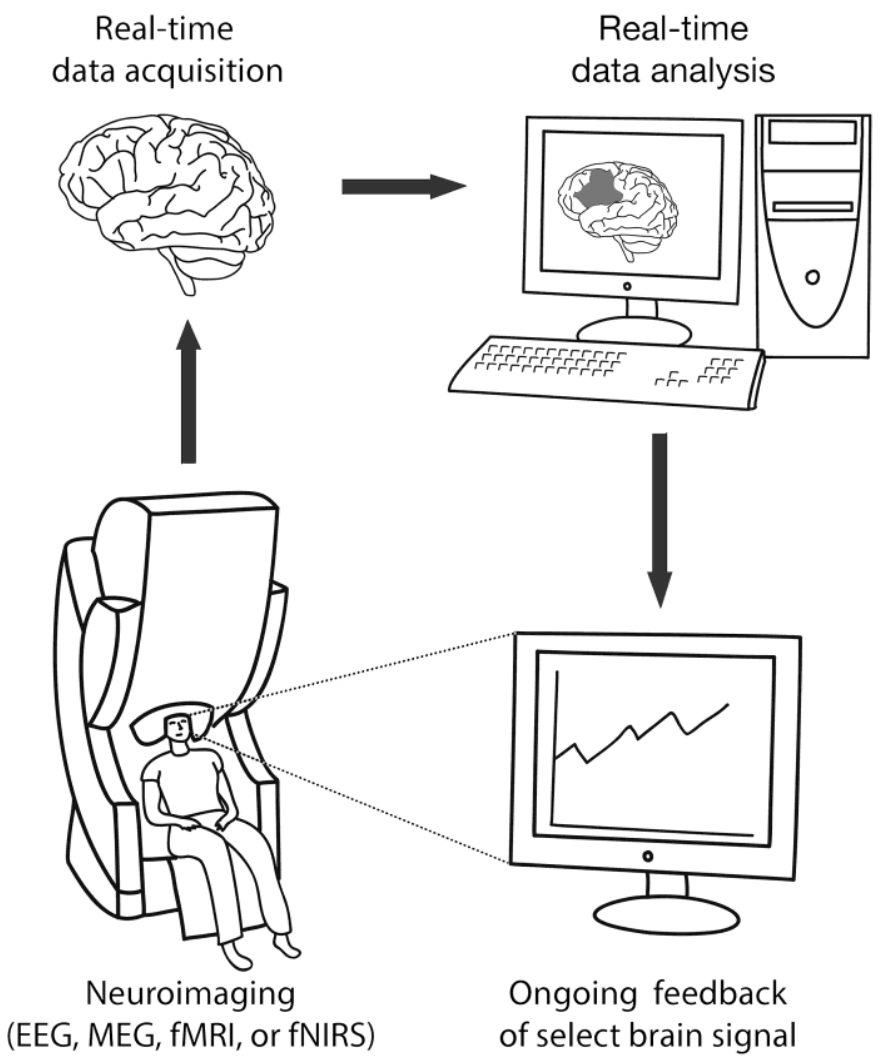

Figure 1. A conceptual diagram of neurofeedback 


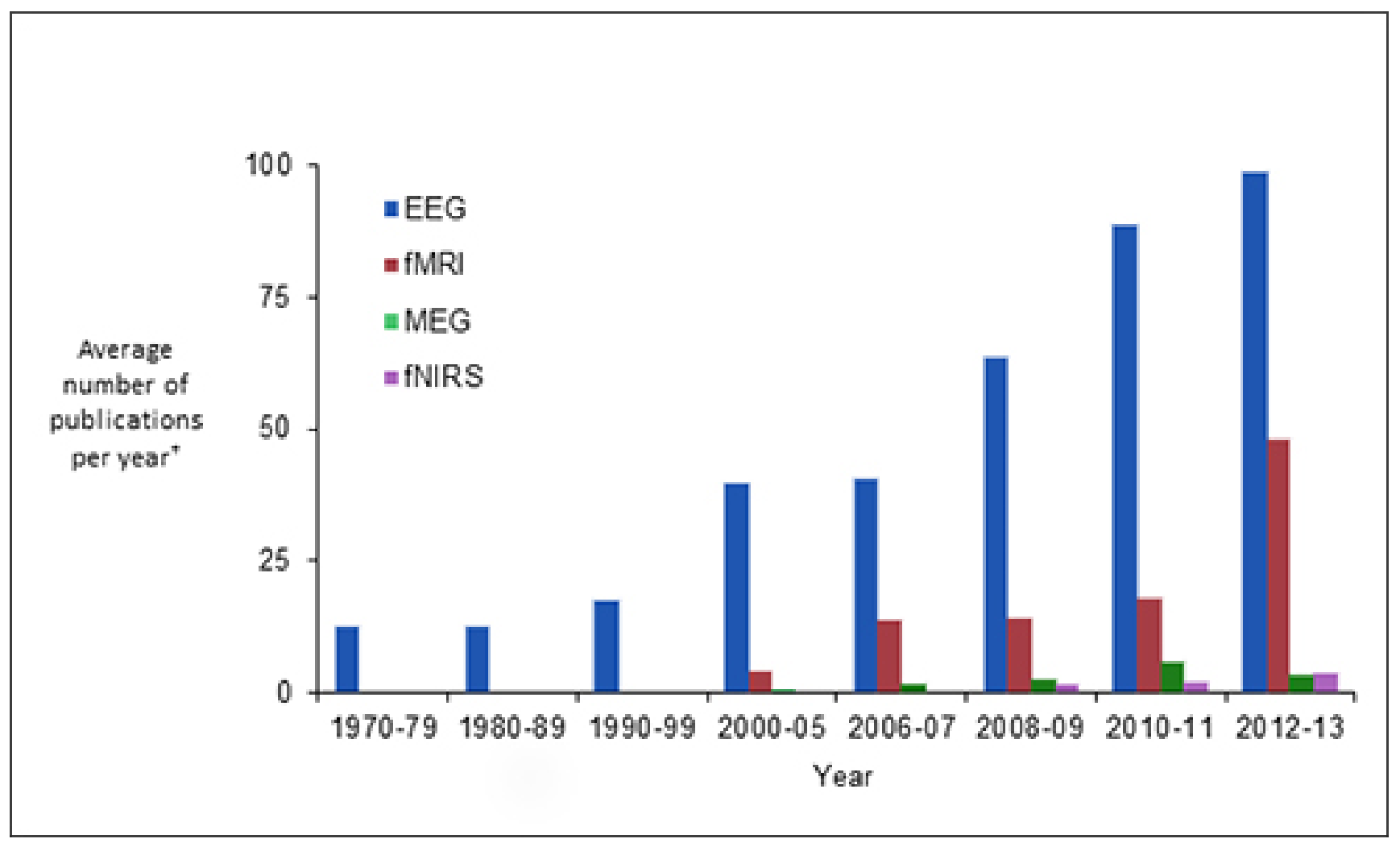

Figure 2. Proliferation of scientific papers on neurofeedback. While EEG-nf research has increased steadily over the past four decades, reports of fMRI-nf have surged in recent years. MEG- and fNIRS-nf remain nascent. †We conducted four distinct literature searches to create this figure. In the topic field of the Web of Science ${ }^{\mathrm{TM}}$ search engine, we entered the keywords EEG or electroencephalograph*, fMRI or "functional magnetic resonance imaging", MEG or magnetoencephalograph*, or fNIRS or "near-infrared spectroscopy". We combined these search terms with the keywords biofeedback OR neurofeedback using the AND operator (e.g., (fMRI OR "functional magnetic resonance imaging") AND (biofeedback OR neurofeedback)). 
IMAGING SCAN

- Alternating activation and rest blocks

- Lying supine (fMRI)

- Sitting upright* (EEG, fNIRS, MEG)

- Approximately 30 minutes

- Few sessions (fMRI) or many (EEG)

\section{Combination treatments}

- Occasionally used

- Offline rehearsal of

mental techniques

- Behavioural therapy

- Medication

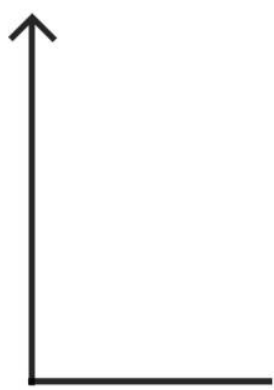

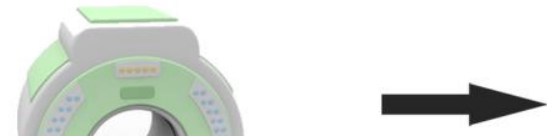

ParTicipants

- 5-30 per study

- Healthy or patient

- Children often used for EEG-nf

- Adults for fMRI-, MEG-, and fNIRS-nf

- Often given mental techniques
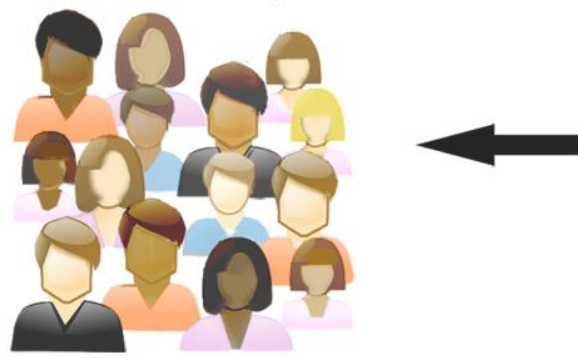

Data Processing

- EEG-nf (many commercial softwares)

- fMRI-nf (Turbo-BrainVoyager or in-house scripts)

- fNIRS, MEG (in-house scripts)

- Usually targets only one signal
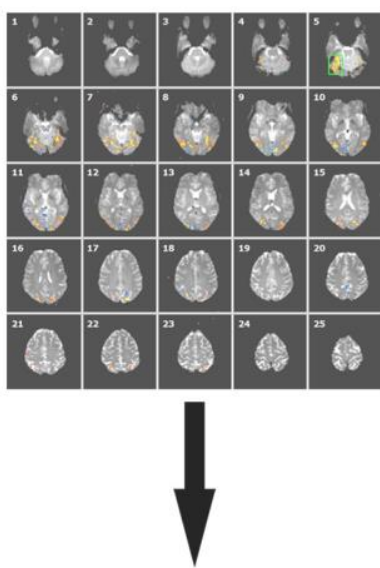

Presentation

- A simple interactive game (EEG)

- Line graph or thermometer (fMRI, fNIRS, MEG)

- Occasionally auditory feedback
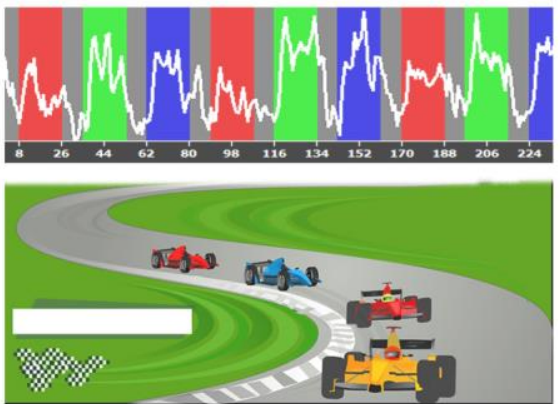

Figure 3. Neurofeedback protocols are diverse and numerous. Participants, brain scanners, data analyses, and feedback presentations vary widely among experiments. *Although rare, individuals can also train EEG-, fNIRS-, and MEG-nf while lying supine or sitting reclined. 


\begin{tabular}{|c|c|c|c|c|}
\hline & EEG & fMRI & MEG & fNIRS \\
\hline $\begin{array}{l}\text { First application to } \\
\text { neurofeedback }\end{array}$ & 1958 & 2003 & 2005 & 2007 \\
\hline $\begin{array}{l}\text { Number of } \\
\text { practitioners } \\
\text { worldwide }\end{array}$ & $>1000$ & None & None & None \\
\hline $\begin{array}{l}\text { Number of } \\
\text { research labs } \dagger\end{array}$ & $>50$ & $\sim 10$ & 3 & $\sim 5$ \\
\hline $\begin{array}{l}\text { Cost of initial } \\
\text { set-up }\end{array}$ & $\begin{array}{l}\$ 500-5,000 \\
\text { (Personal use) } \\
\$ 5,000-50,000 \\
\text { (Research use) }\end{array}$ & $\$ 500,000-2 \mathrm{M}$ & $\$ 2 \mathrm{M}$ & $\begin{array}{l}\$ 50,000- \\
300,000\end{array}$ \\
\hline Running costs $\ddagger$ & No extra fees & $\sim \$ 500 /$ hour & $\sim 500 /$ hour & No extra fees \\
\hline Cost for patient ${ }^{\S}$ & $\begin{array}{l}\$ 130-225 / \text { session } \\
\$ 4,000-10,000 / \\
\text { complete regimen }\end{array}$ & $\begin{array}{l}\text { Not available to } \\
\text { patients }\end{array}$ & $\begin{array}{l}\text { Not available to } \\
\text { patients }\end{array}$ & $\begin{array}{l}\text { Not available to } \\
\text { patients }\end{array}$ \\
\hline $\begin{array}{l}\text { Marketed } \\
\text { equipment }\end{array}$ & $\begin{array}{l}\text { Many companies } \\
\text { sell products for } \\
\text { clinical, research, } \\
\text { and personal uses }\end{array}$ & $\begin{array}{l}\text { One software } \\
\text { package for } \\
\text { research use only }\end{array}$ & $\begin{array}{l}\text { None } \\
\text { (all labs run in- } \\
\text { house software) }\end{array}$ & $\begin{array}{l}\text { None } \\
\text { (all labs run in- } \\
\text { house software) }\end{array}$ \\
\hline
\end{tabular}

Table 1. Popularity, cost, and availability of neurofeedback modalities. Currently, practitioners leverage only EEG-nf in the clinic. Alternatively, fMRI-, MEG-, and fNIRS-nf are costly and lack evidence for clinical application. All prices are in USD. $\uparrow$ This number does not including research labs dedicated solely to BCI research. $\ddagger$ Running costs based on fees charged at the Montreal Neurological Institute in Canada. §Prices vary between centers. We report a representative price taken from an EEG-nf clinic in Austin, Texas and one in New York City. 


\begin{tabular}{|c|c|c|c|}
\hline Training protocol & Target neural signature & $\begin{array}{l}\text { Common } \\
\text { applications }\end{array}$ & $\begin{array}{l}\text { Principal evidence } \\
\text { (observable changes) }\end{array}$ \\
\hline Theta/beta & $\begin{array}{l}\uparrow \text { subset of } 12-21 \mathrm{~Hz} \\
(12-15 \mathrm{~Hz} \text { is SMR training) } \\
\downarrow 4-8 \mathrm{~Hz}\end{array}$ & $\begin{array}{l}\text { ADHD, } \\
\text { epilepsy }\end{array}$ & $\begin{array}{l}\text { ADHD rating scales } \\
\text { Seizure frequency } \\
\text { Resting-state EEG } \\
\text { Resting-state fMRI }\end{array}$ \\
\hline $\begin{array}{l}\text { SCP (slow cortical } \\
\text { potentials) }\end{array}$ & $\begin{array}{l}\uparrow \text { and } \downarrow \text { resting state } \\
\text { electronegativity }\end{array}$ & $\begin{array}{l}\text { ADHD, } \\
\text { epilepsy }\end{array}$ & $\begin{array}{l}\text { Subjective Measures } \\
\text { Resting-state EEG } \\
\text { Resting-state fMRI } \\
\text { Seizure Frequency }\end{array}$ \\
\hline Upper/peak alpha & $\begin{array}{l}\uparrow \text { alpha frequency that is } \\
\text { already largest in amplitude } \\
\text { (often } 9-11 \mathrm{~Hz} \text { ) }\end{array}$ & $\begin{array}{l}\text { Athletic and } \\
\text { cognitive } \\
\text { performance }\end{array}$ & Cognitive tasks \\
\hline $\begin{array}{l}\text { LORETA (low } \\
\text { resolution } \\
\text { electromagnetic } \\
\text { tomography) }\end{array}$ & $\begin{array}{l}\uparrow \text { or } \downarrow \text { activity of select brain } \\
\text { regions (often the anterior } \\
\text { cingulate) }\end{array}$ & $\begin{array}{l}\text { Cognitive } \\
\text { enhancement }\end{array}$ & Intelligence tests \\
\hline Theta + alpha & $\uparrow 4-13 \mathrm{~Hz}$ & Alcoholism & Prevention of relapse \\
\hline Theta/alpha & $\begin{array}{l}\uparrow 4-8 \mathrm{~Hz} \\
\downarrow 8-13 \mathrm{~Hz}\end{array}$ & Creativity & Artistic performance \\
\hline \multicolumn{4}{|c|}{ Commercial Techniques } \\
\hline Live z-score & $\begin{array}{l}\text { Normalize the amplitude and } \\
\text { coherence of all waveforms } \\
\text { (visual and auditory feedback) }\end{array}$ & Any disorder & Subjective measures \\
\hline $\begin{array}{l}\text { LENS (the low } \\
\text { energy } \\
\text { neurofeedback } \\
\text { system) }\end{array}$ & $\begin{array}{l}\text { Normalize the amplitude and } \\
\text { coherence of all waveforms } \\
\text { (electrical pulse feedback) }\end{array}$ & Any disorder & Subjective measures \\
\hline
\end{tabular}

Table 2. Common EEG-nf protocols and applications 